\title{
Zur Besprechung erhaltene Bücher
}

Dürr, W., und K. Kleibohm

Operations Research

Lineare Modelle und ihre

Anwendungen

Studienbücher der Wirtschaft

Carl Hanser, München-Wien 1983.

Schneider, H.-J. (Hrsg.)

Lexikon der Informatik und Daten-

verarbeitung

Oldenbourg, München 1983.
Koch, $\mathrm{H}$.

Integrierte Unternehmensplanung

Gabler, Wiesbaden 1982.

Weber, $\mathrm{M}$.

Entscheidungen bei Mehrfachzielen

Verfahren zur Unterstïtzung von Indi-

vidual- und Gruppenentscheidungen

Bochumer Beiträge zur Unternehmungs-

führung und Unternehmensforschung;

Bd. 26

Gabler, Wiesbaden 1983.

Diese Bücher sind zur Besprechung bei der „Redaktion ZOR“ erhältlich.

\section{Buchbesprechungen}

Bitz, M.: Entscheidungstheorie. Verlag Franz Vahlen, München 1981, XIII + 439 S., DM 64.-.

Dieses Buch kann die Herkunft von den Hagener Fernstudien nicht leugnen: Sehr breite Darstellung, zusammenfassende Wiederholungen und Kontrollfragen am Ende jedes Abschnittes, Úbungsaufgaben mit Lösungen kennzeichnen Stil und Aufbau. Auch der Referenz-Apparat ist sehr sorgfältig gearbeitet: Neben dem Sachverzeichnis findet man ein ausführliches Glossar aller wichtigen, in diesem Buch eingefïhrten Begriffe und schließlich ein Verzeichnis der Abbildungen, die sehr instruktiv und übersichtlich gestaltet sind. Alles in allem ein Buch, ausgezeichnet geeignet für das Selbststudium; der Fachmann mag vielleicht an manchen Stellen etwas mehr Tempo und Stringenz der Schreibeweise wünschen.

Der Inhalt in Stichworten: Grundbegriffe der Entscheidungstheorie, Entscheidungsregeln; Entscheidungsprinzipien für Risikosituationen, insbesondere die klassischen Verteilungscharakteristika, Portefeuilletheorie und Weiterführung zum allgemeinen Bernoulli-Prinzip; Spieltheorie inclusive Nichtnullsummenspiele und einfachste Grundbegriffe der $n$-Personenspiele; schließlich mehrperiodige Entscheidungstheorie mit dem Fisher-Hirshleifer-Modell im Zentrum.

Einige Besonderheiten der Darstellung verdienen hervorgehoben zu werden. Die Systematik der Entscheidungsregeln wird sehr breit - für meinen Geschmack fast etwas zu breit - behandelt, indem gleichsam eine Genesis der Entscheidungsregeln, ausgehend von einer allgemeinen Beschreibung der Formalstruktur von Entscheidungsregeln bei Unsicherheitskonflikten. In der klassischen Risikotheorie wird das $\mu$ - $\sigma$-Prinzip stark herausgehoben; in der Bernoullitheorie des Risikonutzens finden wir in Abschnitt 4.3.3 den bemerkenswerten Versuch einer Aufspaltung dieses Begriffes in einen ,originären Geldnutzen“" und eine „reine Risikopräferenz“. Sehr gut das souveräne Resumé über Zweipersonen-Nichtnullsummenspiele auf S. 256, am Ende des einschlägigen Abschnitts.

Von den an sich selten auftretenden, kleineren Unzukömmlichkeiten seien zwei erwähnt: Eine nicht ganz korrekte Formel (S. 32, (1.06)) für die allgemeine Abhängigkeit des Präferenzwertes einer Alternative von den Ergebnisverteilungen; die Nichtverwendung des Jacobi-Symbols für partielle Ableitungen, speziell bei der Diskussion von Eigenschaften der intertemporalen Präferenzfunktionen (S. 300f.). Schließlich sollte eine Darstellung dieses Umfangs auch durch den Hinweis auf Gegenstände charakterisiert werden, die nicht in den Kreis der Betrachtung gezogen werden. Hier sind es die Entscheidungsfunktionen bzw. die Verarbeitung von Informationen im Falle von Risiko- und Ungewißheitssituationen. Vielleicht mag dies dem Statistiker besonders auffallen, der gewöhnt ist, diesen Aspekt als den für ihn wichtigsten Aspekt der Entscheidungstheorie anzusehen. Dieser Hinweis läßt jedoch den Wert der vorliegenden Darstellung für den Wirtschaftswissenschaftler, insbesondere den Betriebswirt, praktisch unberührt. Alles in allem: Ein Lehrbuch von Rang, das auch dem Kenner an zahlreichen Stellen Anregungen beiten kann. 